\title{
Option Pricing Model with Transaction Costs and Jumps in Illiquid Markets
}

\author{
Praewnapa Seelama ${ }^{1}$, Dawud Thongtha ${ }^{2}$ \\ ${ }^{1}$ Department of Mathematics, Faculty of Science, King Mongkut's University of Technology Thonburi (KMUTT), Thung Khru, \\ Bangkok, Thailand \\ ${ }^{2}$ Mathematics and Statistics with Applications (MaSA), Department of Mathematics, Faculty of Science, King Mongkut's \\ University of Technology Thonburi (KMUTT), Thung Khru, Bangkok, Thailand \\ Email: praewnapa.jar@mail.kmutt.ac.th,dawud.tho@kmutt.ac.th
}

How to cite this paper: Seelama, P. and Thongtha, D. (2021) Option Pricing Model with Transaction Costs and Jumps in Illiquid Markets. Journal of Mathematical Finance, 11, 361-372.

https://doi.org/10.4236/jmf.2021.113020

Received: March 31, 2021

Accepted: June 7, 2021

Published: June 10, 2021

Copyright (c) 2021 by author(s) and Scientific Research Publishing Inc. This work is licensed under the Creative Commons Attribution International License (CC BY 4.0).

http://creativecommons.org/licenses/by/4.0/ (cc) (i) Open Access

\begin{abstract}
Option pricing model is a wildly interested topic in an area of financial Mathematics. The pioneer model was introduced by Fischer Black and Myron Scholes which is known as the Black-Scholes model. This model was derived under various assumptions such as liquidity and no transaction costs for which a underlying asset price in stock market might not be satisfied. With this fact, the underlying asset price models were remodeled, in order to determine an option value. This research aims to extend the Black-Scholes model by relaxing the assumption of no transaction costs in illiquid markets. Also, jumps of asset price are considered in this work. To do this, a differential form of asset price with transaction costs and jumps in illiquid markets is introduced and then used to construct the extended option pricing model. Furthermore, a numerical result of a call option price under a new situation is provided.
\end{abstract}

\section{Keywords}

Option Pricing, The Black-Scholes Model, Jump Diffusion Model, Illiquid Markets, Transaction Costs

\section{Introduction}

Derivatives are financial instruments that give the right to buy or sell an underlying asset in the future. These contacts, such as future, forward, swap and option, were used for speculating and managing risks in an investment. For an option, it is a financial contract that gives option holders the right to buy or sell an underlying asset from option writers by a specified date and price. The contract, 
giving option holders the right to buy an underlying asset, is called a call option while the contract that gives option holders the right to sell an underlying asset is called a put option.

In 1973, Fischer Black and Myron Scholes [1] constructed the Black-Scholes model for determining prices of options. However, their model required various assumptions such as constant volatility, no transaction costs and perfect liquidity. However, purchasing on some stocks may be illiquid. Also, random jumps of prices of some underlying assets have occurred. With these reasons, the Black-Scholes assumptions may not fulfill the real financial market situations. Therefore, many researchers tried to extend the result of Black and Scholes by reducing some of the above assumptions (see, [2] [3] and [4]).

In illiquid market, the investor's trading in the stock market affects the stock price. This impact is called a price impact. The price impact is referred to the correlation between trading and subsequent price change. This may be a result from a bid trader who is able to move the price by his/her actions.

In 2005, Hong Liu and Jiongmin Yong [5] examined the effects of price impact in an illiquid market in replicating a European option. They investigated a generalized Black-Scholes pricing model in illiquid market. Moreover, the presence of the price impact has been studied and analysed in several researches. For example, Kristoffer Glover, Peter Duck, and David Newton [6] consider the effects of illiquidity on the Black-Scholes model. Traian Pirvu and Ahmadreza Yazdanian [7] investigated the effects of price impact in imperfect liquidity on the replication of a European Spread option.

In 2013, Youssef El-Khatib and Abdulnasser Hatemi-J [8] applied a jump diffusion model to price process in Liu and Yong [5] and provided the Black-Scholes model in illiquid with Jump. Then, in 2016, Francis Agana, Oluwole D. Makinde and David M. Theuri [9] studied the combined effects of transaction costs and large trading in illiquid markets on options pricing model. They derived a generalized and nonlinear Black-Scholes model in an illiquid financial market with transaction costs. However, none of the previous research is studied under the situation of existing transaction costs and jumps associated with illiquid markets.

In this paper, we combined the idea of [8] and [9] by introducing a differential form of an asset price process related to transaction costs and jump diffusion term in an illiquid market. Also, we provide a European option pricing model with transaction costs and jumps in illiquid markets. This model extends results in [1] [5] [8] and [9] by reducing more assumptions. Moreover, numerical simulations of an option price are shown by using the Monte Carlo simulation.

The contents of this research are organized into four sections. In Section 2, the differential form of assets price with transaction costs and jumps in illiquid markets is introduced. Also, an asset price process is investigated and a simulation example of option price is given in this section. In Section 3, a model of option pricing associated to the propose differential form is provided. Finally, con- 
cluding remarks are given in Section 4.

\section{Differential Form of Assets Price with Transaction Costs and Jumps in Illiquid Market}

In this section, we introduce the differential form of assets price with transactions costs and jumps for illiquid market. We consider a financial market having two types of assets; a risk-free asset and a risky asset. For $t>0$, let $A_{t}$ and $S_{t}$ be risk-free asset and risky asset prices at time $t$, respectively, $T-t$ be the time to maturity date, $K$ be strike price and $h\left(S_{T}\right)=\left(S_{T}-K\right)^{+}=\max \left\{S_{T}-K, 0\right\}$ be the pay-off at time $T$. In 1973, Fischer Black and Myron Scholes [1] constructed the Black-Scholes model for option pricing. They assumed that the price of risk-free asset follows

$$
\mathrm{d} A_{t}=r A_{t} \mathrm{~d} t
$$

where $r$ is the risk-free interest rate and assumed that the price of risky asset satisfies

$$
\mathrm{d} S_{t}=S_{t}\left(\mu \mathrm{d} t+\sigma \mathrm{d} W_{t}\right)
$$

where $\mu$ and $\sigma$ are the constant drift and constant volatility, respectively, $W_{t}$ is a standard one-dimensional Brownian motion. In 2005, Hong Liu and Jiongmin Yong [5] extended the result of Black and Scholes [1]. They derived a generalized Black-Scholes pricing model in illiquid market. In [5], Hong Liu and Jiongmin Yong assumed that the price of risk-free asset and the price of risky asset follow

$$
\begin{gathered}
\mathrm{d} A_{t}=r\left(t, S_{t}\right) A_{t} \mathrm{~d} t \text { and } \\
\mathrm{d} S_{t}=S_{t}\left(\mu\left(t, S_{t}\right) \mathrm{d} t+\sigma\left(t, S_{t}\right) \mathrm{d} W_{t}+\lambda\left(t, S_{t}\right) \mathrm{d} \theta_{t}\right)
\end{gathered}
$$

where $r\left(t, S_{t}\right)$ is the interest rate, the drift and the volatility, respectively, depending on time $t$ and $S_{t}, \lambda\left(t, S_{t}\right)$ is price impact function of the trader (non-negative) and $\theta_{t}$ is the number of shares. They also assumed that

$$
\mathrm{d} \theta_{t}=\eta_{t} \mathrm{~d} t+\zeta_{t} \mathrm{~d} W_{t}
$$

where $\eta_{t}$ and $\zeta_{t}$ are adapted process to a filtration $\left(\mathcal{F}_{t}\right)_{t>0}$ generated by the Brownian motion. After that, in 2013, Youssef El-Khatib and Abdulnasser Hatemi-J [8] applied a jump diffusion model to price process in Liu and Yong model [5]. The price of risky asset is assumed as

$$
\mathrm{d} S_{t}=S_{t}\left(\mu\left(t, S_{t}\right) \mathrm{d} t+\sigma\left(t, S_{t}\right)\left(\mathrm{d} W_{t}+a \mathrm{~d} M_{t}\right)+\lambda\left(t, S_{t}\right) \mathrm{d} \theta_{t}\right)
$$

where $a$ is a real constant and $M_{t}=N_{t}-\rho t$ is the compensated Poisson process where $N_{t}$ is a Poisson process with deterministic intensity $\rho$. They assumed further that

$$
\mathrm{d} \theta_{t}=\eta_{t} \mathrm{~d} t+\zeta_{t}\left(\mathrm{~d} W_{t}+b \mathrm{~d} M_{t}\right)
$$

where $b$ is a real constant. In 2016, Francis Agana and et al. [9] added the term 
of transaction costs to price process in Liu and Yong model [5]. They assumed that the price of risky asset satisfies

$$
\mathrm{d} S_{t}=S_{t}\left(\mu\left(t, S_{t}\right) \mathrm{d} t+\sigma\left(t, S_{t}\right) \mathrm{d} W_{t}+\lambda\left(t, S_{t}\right) \mathrm{d} \theta_{t}+\kappa\left(t, S_{t}\right) \mathrm{d} \theta_{t}\right)
$$

where $\kappa\left(t, S_{t}\right)$ is the transaction costs. In this work, we combined the idea of [8] and [9] to construct a model of option pricing. We assume that the price of the risky asset is generated by the following stochastic differential equation:

$$
\mathrm{d} S_{t}=S_{t}\left(\mu\left(t, S_{t}\right) \mathrm{d} t+\sigma\left(t, S_{t}\right)\left(\mathrm{d} W_{t}+a \mathrm{~d} M_{t}\right)+\lambda\left(t, S_{t}\right) \mathrm{d} \theta_{t}+\kappa\left(t, S_{t}\right) \mathrm{d} \theta_{t}\right)
$$

and $\theta_{t}$ satisfies

$$
\mathrm{d} \theta_{t}=\eta_{t} \mathrm{~d} t+\zeta_{t}\left(\mathrm{~d} W_{t}+b \mathrm{~d} M_{t}\right)
$$

Thus, by (9) and (10), the price process of the risky asset satisfies the following differential form:

$$
\begin{aligned}
\mathrm{d} S_{t}= & S_{t}\left(\left[\mu\left(t, S_{t}\right)+\lambda\left(t, S_{t}\right) \eta_{t}+\kappa\left(t, S_{t}\right) \eta_{t}\right] \mathrm{d} t\right. \\
& +\left[\sigma\left(t, S_{t}\right)+\lambda\left(t, S_{t}\right) \zeta_{t}+\kappa\left(t, S_{t}\right) \zeta_{t}\right] \mathrm{d} W_{t} \\
& \left.+\left[a \sigma\left(t, S_{t}\right)+b \lambda\left(t, S_{t}\right) \zeta_{t}+b \kappa\left(t, S_{t}\right) \zeta_{t}\right] \mathrm{d} M_{t}\right) .
\end{aligned}
$$

In solving Equation (11), we apply the Ito lemma in [8] with $G\left(t, S_{t}\right)=\ln S_{t}$. We obtain

$$
\begin{aligned}
\ln S_{t}= & \ln S_{0}+\int_{0}^{t}\left[\partial_{s} \ln S_{s}+\left(\mu\left(s, S_{s}\right)+\lambda\left(s, S_{s}\right) \eta_{s}+\kappa\left(s, S_{s}\right) \eta_{s}\right.\right. \\
& \left.-\rho\left[a \sigma\left(s, S_{s}\right)+b \lambda\left(s, S_{s}\right) \zeta_{s}+b \kappa\left(s, S_{s}\right) \zeta_{s}\right]\right) S_{s} \partial_{x} \ln S_{s} \\
& +\frac{1}{2}\left(\sigma\left(s, S_{s}\right)+\lambda\left(s, S_{s}\right) \zeta_{s}+\kappa\left(s, S_{s}\right) \zeta_{s}\right)^{2} S_{s}^{2} \partial_{x x}^{2} \ln S_{s} \\
& \left.+\rho\left(\ln \left(S_{s^{-}}+\left(a \sigma\left(s, S_{s}\right)+b \lambda\left(s, S_{s}\right) \zeta_{s}+b \kappa\left(s, S_{s}\right) \zeta_{s}\right) S_{s}\right)-\ln S_{s^{-}}\right)\right] \mathrm{d} s \\
& +\int_{0}^{t}\left(\sigma\left(s, S_{s}\right)+\lambda\left(s, S_{s}\right) \zeta_{s}+\kappa\left(s, S_{s}\right) \zeta_{s}\right) S_{s} \partial_{x} \ln S_{s} \mathrm{~d} W_{s} \\
& +\int_{0}^{t}\left(\ln \left(S_{s^{-}}+\left(a \sigma\left(s, S_{s}\right)+b \lambda\left(s, S_{s}\right) \zeta_{s}+b \kappa\left(s, S_{s}\right) \zeta_{s}\right) S_{s}\right)-\ln S_{s^{-}}\right) \mathrm{d} M_{s} \\
= & \ln S_{0}+\int_{0}^{t}\left[\left(\mu\left(s, S_{s}\right)+\lambda\left(s, S_{s}\right) \eta_{s}+\kappa\left(s, S_{s}\right) \eta_{s}\right.\right. \\
& \left.-\rho\left[a \sigma\left(s, S_{s}\right)+b \lambda\left(s, S_{s}\right) \zeta_{s}+b \kappa\left(s, S_{s}\right) \zeta_{s}\right]\right) S_{s} \frac{1}{S_{s}} \\
& -\frac{1}{2}\left(\sigma\left(s, S_{s}\right)+\lambda\left(s, S_{s}\right) \zeta_{s}+\kappa\left(s, S_{s}\right) \zeta_{s}\right)^{2} S_{s}^{2} \frac{1}{S_{s}^{2}} \\
& \left.+\ln S_{s^{-}}\right)\left(\mathrm{d} N_{s}-\rho \mathrm{d} s\right) \\
+ & \int_{0}^{t}\left(\sigma\left(s, S_{s}\right)+\lambda\left(s, S_{s}\right) \zeta_{s}+\kappa\left(s, S_{s}\right) \zeta_{s}\right) S_{s} \frac{1}{S_{s}} \mathrm{~d} W_{s} \\
& \left.\left.\left.\left(1+a \sigma\left(s, S_{s}\right)+b \lambda\left(s, S_{s}\right) \zeta_{s}+b \kappa\left(s, S_{s}\right) \zeta_{s}\right)\right)-\ln S_{s^{-}}\right)\right] \mathrm{d} s \\
& \\
&
\end{aligned}
$$




$$
\begin{aligned}
= & \ln S_{0}+\int_{0}^{t}\left[\left(\mu\left(s, S_{s}\right)+\lambda\left(s, S_{s}\right) \eta_{s}+\kappa\left(s, S_{s}\right) \eta_{s}\right.\right. \\
& \left.-\rho\left[a \sigma\left(s, S_{s}\right)+b \lambda\left(s, S_{s}\right) \zeta_{s}+b \kappa\left(s, S_{s}\right) \zeta_{s}\right]\right) \\
& \left.-\frac{1}{2}\left(\sigma\left(s, S_{s}\right)+\lambda\left(s, S_{s}\right) \zeta_{s}+\kappa\left(s, S_{s}\right) \zeta_{s}\right)^{2}\right] \mathrm{d} s \\
& +\int_{0}^{t}\left(\sigma\left(s, S_{s}\right)+\lambda\left(s, S_{s}\right) \zeta_{s}+\kappa\left(s, S_{s}\right) \zeta_{s}\right) \mathrm{d} W_{s} \\
& +\int_{0}^{t} \ln \left(1+a \sigma\left(s, S_{s}\right)+b \lambda\left(s, S_{s}\right) \zeta_{s}+b \kappa\left(s, S_{s}\right) \zeta_{s}\right) \mathrm{d} N_{s}
\end{aligned}
$$

The Poisson integral in (12) can be computed in terms of the waiting times $T_{k}$. Thus, we have

$$
\begin{aligned}
\ln S_{t}= & \ln S_{0}+\int_{0}^{t}\left[\left(\mu\left(s, S_{s}\right)+\lambda\left(s, S_{s}\right) \eta_{s}+\kappa\left(s, S_{s}\right) \eta_{s}\right.\right. \\
& \left.-\rho\left[a \sigma\left(s, S_{s}\right)+b \lambda\left(s, S_{s}\right) \zeta_{s}+b \kappa\left(s, S_{s}\right) \zeta_{s}\right]\right) \\
& \left.-\frac{1}{2}\left(\sigma\left(s, S_{s}\right)+\lambda\left(s, S_{s}\right) \zeta_{s}+\kappa\left(s, S_{s}\right) \zeta_{s}\right)^{2}\right] \mathrm{d} s \\
& +\int_{0}^{t}\left(\sigma\left(s, S_{s}\right)+\lambda\left(s, S_{s}\right) \zeta_{s}+\kappa\left(s, S_{s}\right) \zeta_{s}\right) \mathrm{d} W_{s} \\
& +\sum_{k=1}^{N_{t}} \ln \left(1+a \sigma\left(T_{k}, S_{T_{k}}\right)+b \lambda\left(T_{k}, S_{T_{k}}\right) \zeta_{T_{k}}+b \kappa\left(T_{k}, S_{T_{k}}\right) \zeta_{T_{k}}\right) \Delta N_{t}
\end{aligned}
$$

where $\Delta N_{t}=1$, that is the Poisson process has a jump at time $t$. Hence, the asset price $S_{t}$ at time $t$ is given by

$$
\begin{aligned}
S_{t}= & S_{0} \exp \left(\int _ { 0 } ^ { t } \left[\left(\mu\left(s, S_{s}\right)+\lambda\left(s, S_{s}\right) \eta_{s}+\kappa\left(s, S_{s}\right) \eta_{s}\right.\right.\right. \\
& \left.-\rho\left[a \sigma\left(s, S_{s}\right)+b \lambda\left(s, S_{s}\right) \zeta_{s}+b \kappa\left(s, S_{s}\right) \zeta_{s}\right]\right) \\
& \left.-\frac{1}{2}\left(\sigma\left(s, S_{s}\right)+\lambda\left(s, S_{s}\right) \zeta_{s}+\kappa\left(s, S_{s}\right) \zeta_{s}\right)^{2}\right] \mathrm{d} s \\
& \left.+\int_{0}^{t}\left(\sigma\left(s, S_{s}\right)+\lambda\left(s, S_{s}\right) \zeta_{s}+\kappa\left(s, S_{s}\right) \zeta_{s}\right) \mathrm{d} W_{s}\right) \\
& \times \prod_{k=1}^{N_{t}}\left(1+a \sigma\left(T_{k}, S_{T_{k}}\right)+b \lambda\left(T_{k}, S_{T_{k}}\right) \zeta_{T_{k}}+b \kappa\left(T_{k}, S_{T_{k}}\right) \zeta_{T_{k}}\right)
\end{aligned}
$$

Next, a Monte Carlo simulation for a call option price is presented. This computation is obtained as a special case when the coefficients $\mu, \lambda, \kappa, \eta, \zeta, a, b$ and $\rho$ are constants. By Equation (14), the stock price at maturity date $T$ can be simplified as

$$
\begin{aligned}
S_{T}= & S_{0} \exp \left(\left(\mu+\lambda \eta+\kappa \eta-\rho(a \sigma+b \lambda \zeta+b \kappa \zeta)-\frac{1}{2}(\sigma+\lambda \zeta+\kappa \zeta)^{2}\right) T\right. \\
& \left.+(\sigma+\lambda \zeta+\kappa \zeta) W_{T}\right) \times(1+a \sigma+b \lambda \zeta+b \kappa \zeta)^{N_{T}}
\end{aligned}
$$

and the price of a call option with strike price $K$ at maturity date $T$ is computed as 


$$
E\left[\mathrm{e}^{-r T}\left(S_{T}-K\right)^{+}\right]
$$

If we set $S_{0}=40, r=0.05, \mu=0.02, \sigma=0.2, K=44, \lambda=0.0001$, $\kappa=0.01, \eta=0.001, \zeta=0.05, a=0.1, \quad b=0.3$ and $\rho=1$, the simulation results are shown below. In Figure 1, a Monte Carlo simulation result of asset price followed the Equation (15) with $T=1$, the number of time steps $m=100$ and the number of realizations $n=500$ is shown. The average of the asset price from the simulation result is depicted in Figure 2. In Figure 3, a call option price in Equation (16) is simulated.

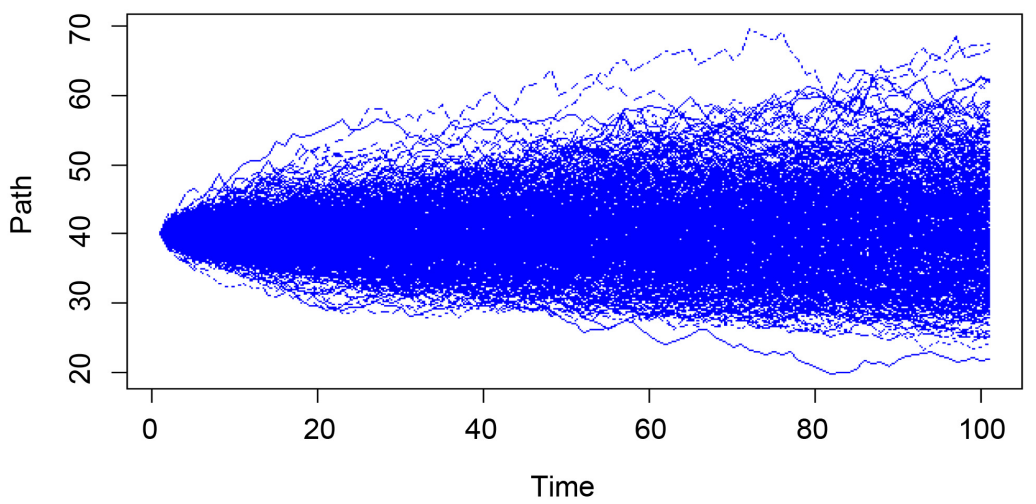

Figure 1. The simulation of the asset price.

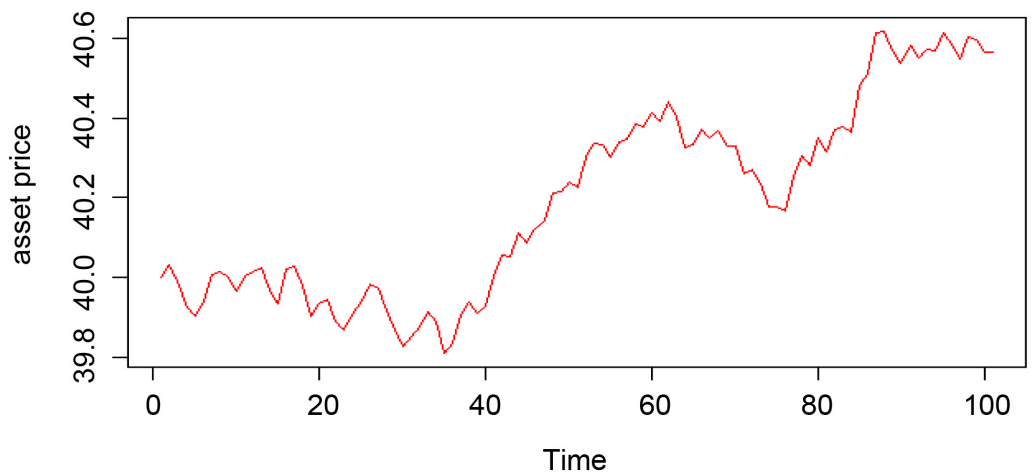

Figure 2. The average of the asset price.

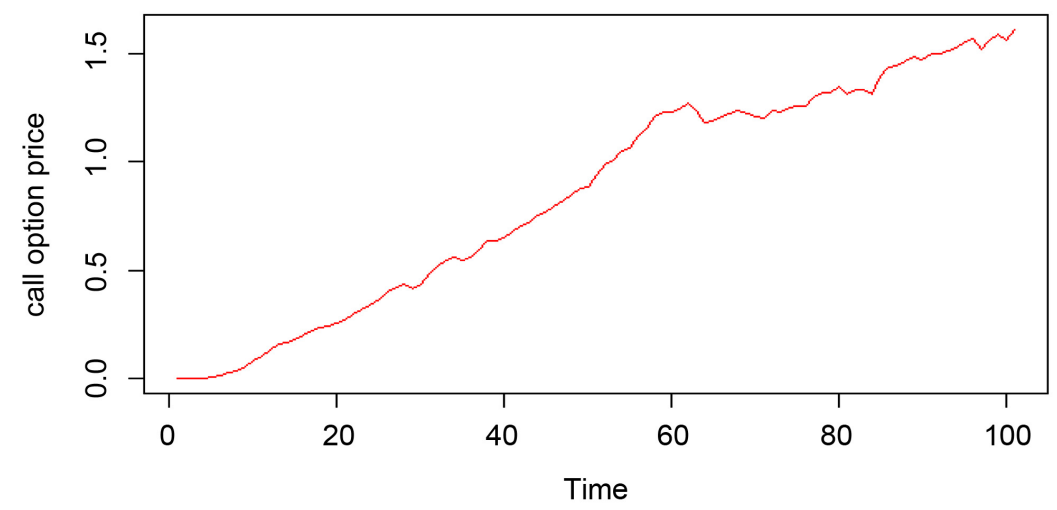

Figure 3. The simulation of European call option price. 
It can be seen form the above figures that there are wide ranges of values in a number of paths being result form jumps, transaction costs and price impacts. However, the average of the asset price lies between 39 and 41. Although the simulated price at expiration date is less than the strike price which is 44 , the simulated option price is still positive due to the definition of pay-off. This can be explained that if an investor plans to buy and then hold an option, which an underlying asset price follows Equation (9), to its expiration date, the option fair price at current time is positive because of positive jumps of the underlying asset price.

\section{Option Pricing Model with Transaction Costs and Jumps in Illiquid Markets}

In this section, we construct a partial differential equation for option pricing by using a arbitrage pricing technique [10] consisting of the following steps:

1) Constructing a self-financing portfolio with the risk-free asset and the risky asset.

2) Providing a differential form of option price by applying Ito's lemma to option price function depending on $t$ and $S_{t}$.

3) Comparing the coefficients in the above differential form by using arbitrage pricing technique. This is, comparing the coefficients in random and non random parts in the replicating portfolio from step 1.

(Step 1) For $t \in[0, T]$, let $V_{t}$ is the wealth process and $\psi_{t}$ is the number of units invested in the risk-free asset. The value of the portfolio $V_{t}$ satisfies

$$
V_{t}=\psi_{t} A_{t}+\theta_{t} S_{t}
$$

Assume that the trading portfolio is self-financing. Then,

$$
\mathrm{d} V_{t}=\psi_{t} \mathrm{~d} A_{t}+\theta_{t} \mathrm{~d} S_{t} .
$$

and we have the following Proposition.

Proposition 1. If the portfolio is self-financing, then the wealth process in (18) follows the stochastic differential equation:

$$
\begin{aligned}
\mathrm{d} V_{t}= & \left(r\left(t, S_{t}\right) V_{t}+\theta_{t} S_{t}\left[\mu\left(t, S_{t}\right)-r\left(t, S_{t}\right)+\lambda\left(t, S_{t}\right) \eta_{t}+\kappa\left(t, S_{t}\right) \eta_{t}\right]\right) \mathrm{d} t \\
& +\theta_{t} S_{t}\left[\sigma\left(t, S_{t}\right)+\lambda\left(t, S_{t}\right) \zeta_{t}+\kappa\left(t, S_{t}\right) \zeta_{t}\right] \mathrm{d} W_{t} \\
& +\theta_{t} S_{t}\left[a \sigma\left(t, S_{t}\right)+b \lambda\left(t, S_{t}\right) \zeta_{t}+b \kappa\left(t, S_{t}\right) \zeta_{t}\right] \mathrm{d} M_{t} .
\end{aligned}
$$

Proof. By (3), (11), (17) and (18), we have

$$
\begin{aligned}
\mathrm{d} V_{t}= & \psi_{t} \mathrm{~d} A_{t}+\theta_{t} \mathrm{~d} S_{t}=\frac{V_{t}-\theta_{t} S_{t}}{A_{t}} \mathrm{~d} A_{t}+\theta_{t} \mathrm{~d} S_{t} \\
= & \frac{V_{t}-\theta_{t} S_{t}}{A_{t}}\left(r\left(t, S_{t}\right) A_{t} \mathrm{~d} t\right)+\theta_{t} S_{t}\left(\left[\mu\left(t, S_{t}\right)+\lambda\left(t, S_{t}\right) \eta_{t}+\kappa\left(t, S_{t}\right) \eta_{t}\right] \mathrm{d} t\right. \\
& +\left[\sigma\left(t, S_{t}\right)+\lambda\left(t, S_{t}\right) \zeta_{t}+\kappa\left(t, S_{t}\right) \zeta_{t}\right] \mathrm{d} W_{t} \\
& \left.+\left[a \sigma\left(t, S_{t}\right)+b \lambda\left(t, S_{t}\right) \zeta_{t}+b \kappa\left(t, S_{t}\right) \zeta_{t}\right] \mathrm{d} M_{t}\right)
\end{aligned}
$$




$$
\begin{aligned}
= & \left(r\left(t, S_{t}\right) V_{t}+\theta_{t} S_{t}\left[\mu\left(t, S_{t}\right)-r\left(t, S_{t}\right)+\lambda\left(t, S_{t}\right) \eta_{t}+\kappa\left(t, S_{t}\right) \eta_{t}\right]\right) \mathrm{d} t \\
& +\theta_{t} S_{t}\left[\sigma\left(t, S_{t}\right)+\lambda\left(t, S_{t}\right) \zeta_{t}+\kappa\left(t, S_{t}\right) \zeta_{t}\right] \mathrm{d} W_{t} \\
& +\theta_{t} S_{t}\left[a \sigma\left(t, S_{t}\right)+b \lambda\left(t, S_{t}\right) \zeta_{t}+b \kappa\left(t, S_{t}\right) \zeta_{t}\right] \mathrm{d} M_{t} .
\end{aligned}
$$

Therefore, the proposition is proved.

The following theorem gives the partial differential equation for option pricing with transaction costs and jumps in illiquid markets. In this theorem, we combine the step 2 and step 3 in arbitrage pricing technique.

Theorem 2. Let $f\left(t, S_{t}\right)$ be the price of the European call option at time $t \in[0, T]$ and $S_{t}$ satisfies the Equation (11). Then the partial differential equation of the option price is given by

$$
\begin{aligned}
& r\left(t, S_{t}\right) V_{t}+\theta_{t} S_{t}\left[\mu\left(t, S_{t}\right)-r\left(t, S_{t}\right)+\lambda\left(t, S_{t}\right) \eta_{t}+\kappa\left(t, S_{t}\right) \eta_{t}\right] \\
& =\partial_{t} f\left(t, S_{t}\right)+\left(\mu\left(t, S_{t}\right)+\lambda\left(t, S_{t}\right) \eta_{t}+\kappa\left(t, S_{t}\right) \eta_{t}\right. \\
& \left.\quad-\rho\left[a \sigma\left(t, S_{t}\right)+b \lambda\left(t, S_{t}\right) \zeta_{t}+b \kappa\left(t, S_{t}\right) \zeta_{t}\right]\right) S_{t} \partial_{S} f\left(t, S_{t}\right) \\
& \quad+\frac{1}{2}\left(\sigma\left(t, S_{t}\right)+\lambda\left(t, S_{t}\right) \zeta_{t}+\kappa\left(t, S_{t}\right) \zeta_{t}\right)^{2} S_{t}^{2} \partial_{S S}^{2} f\left(t, S_{t}\right) \\
& \quad+\rho\left(f\left(t, S_{t^{-}}\left(1+a \sigma\left(t, S_{t}\right)+b \lambda\left(t, S_{t}\right) \zeta_{t}+b \kappa\left(t, S_{t}\right) \zeta_{t}\right)\right)-f\left(t, S_{t^{-}}\right)\right)
\end{aligned}
$$

with the terminal condition $f\left(T, S_{T}\right)=h\left(S_{T}\right)$.

Proof. (Step 2) Let $C^{1,2}([0, T] \times[-\infty, \infty])$ be the continuously differentiable functions. Assume that function $f\left(t, S_{t}\right) \in C^{1,2}([0, T] \times[-\infty, \infty])$, that is the first derivative with respect to $t$ and the first and second derivative respect to $S$ of function $f$ are exist and continuous. Also assumed that $f\left(t, S_{t}\right)$ be the price of a European option and the stock price $S_{t}$ satisfies the Equation (11). By Ito lemma in [8] with $G\left(t, X_{t}\right)=f\left(t, S_{t}\right)$. We obtain the differential form of option price satisfies the following stochastic differential equation:

$$
\begin{aligned}
\mathrm{d} f\left(t, S_{t}\right)= & {\left[\partial_{t} f\left(t, S_{t}\right)+\left(\mu\left(t, S_{t}\right)+\lambda\left(t, S_{t}\right) \eta_{t}+\kappa\left(t, S_{t}\right) \eta_{t}\right.\right.} \\
& \left.-\rho\left[a \sigma\left(t, S_{t}\right)+b \lambda\left(t, S_{t}\right) \zeta_{t}+b \kappa\left(t, S_{t}\right) \zeta_{t}\right]\right) S_{t} \partial_{S} f\left(t, S_{t}\right) \\
& +\frac{1}{2}\left(\sigma\left(t, S_{t}\right)+\lambda\left(t, S_{t}\right) \zeta_{t}+\kappa\left(t, S_{t}\right) \zeta_{t}\right)^{2} S_{t}^{2} \partial_{S S}^{2} f\left(t, S_{t}\right) \\
& +\rho\left(f\left(t, S_{t^{-}}\left(1+a \sigma\left(t, S_{t}\right)+b \lambda\left(t, S_{t}\right) \zeta_{t}+b \kappa\left(t, S_{t}\right) \zeta_{t}\right)\right)\right. \\
& \left.\left.-f\left(t, S_{t^{-}}\right)\right)\right] \mathrm{d} t \\
& +\left(\sigma\left(t, S_{t}\right)+\lambda\left(t, S_{t}\right) \zeta_{t}+\kappa\left(t, S_{t}\right) \zeta_{t}\right) S_{t} \partial_{S} f\left(t, S_{t}\right) \mathrm{d} W_{t} \\
& +\left(f\left(t, S_{t^{-}}\left(1+a \sigma\left(t, S_{t}\right)+b \lambda\left(t, S_{t}\right) \zeta_{t}+b \kappa\left(t, S_{t}\right) \zeta_{t}\right)\right)\right. \\
& \left.-f\left(t, S_{t^{-}}\right)\right) \mathrm{d} M_{t}
\end{aligned}
$$

(Step 3) By arbitrage pricing technique, this implies that $\mathrm{d} V_{t}=\mathrm{d} f\left(t, S_{t}\right)$. Thus, we can compare the coefficients for $d W_{t}$ in Equations (19) and (21), we have 


$$
\begin{aligned}
& \theta_{t} S_{t}\left[\sigma\left(t, S_{t}\right)+\lambda\left(t, S_{t}\right) \zeta_{t}+\kappa\left(t, S_{t}\right) \zeta_{t}\right] \\
& =\left[\sigma\left(t, S_{t}\right)+\lambda\left(t, S_{t}\right) \zeta_{t}+\kappa\left(t, S_{t}\right) \zeta_{t}\right] S_{t} \partial_{S} f\left(t, S_{t}\right)
\end{aligned}
$$

If $\sigma\left(t, S_{t}\right)+\lambda\left(t, S_{t}\right) \zeta_{t}+\kappa\left(t, S_{t}\right) \zeta_{t} \neq 0$, the above equation implies that

$$
\theta_{t}=\partial_{S} f\left(t, S_{t}\right)
$$

Similarly for the coefficient of $\mathrm{d} M_{t}$, we have

$$
\begin{aligned}
& \theta_{t} S_{t}\left[a \sigma\left(t, S_{t}\right)+b \lambda\left(t, S_{t}\right) \zeta_{t}+b \kappa\left(t, S_{t}\right) \zeta_{t}\right] \\
& =f\left(t, S_{t^{-}}\left(1+a \sigma\left(t, S_{t}\right)+b \lambda\left(t, S_{t}\right) \zeta_{t}+b \kappa\left(t, S_{t}\right) \zeta_{t}\right)\right)-f\left(t, S_{t^{-}}\right)
\end{aligned}
$$

which implies that

$$
\theta_{t}=\frac{f\left(t, S_{t^{-}}\left(1+a \sigma\left(t, S_{t}\right)+b \lambda\left(t, S_{t}\right) \zeta_{t}+b \kappa\left(t, S_{t}\right) \zeta_{t}\right)\right)-f\left(t, S_{t^{-}}\right)}{S_{t}\left[a \sigma\left(t, S_{t}\right)+b \lambda\left(t, S_{t}\right) \zeta_{t}+b \kappa\left(t, S_{t}\right) \zeta_{t}\right]}
$$

Consider Equations (22) and (23), we can see that the $\theta_{t}$ in Equation (22) has no jumping variable but that in Equation (23) depends on jump. Hence, by comparing the coefficients of $\mathrm{d} W_{t}$ and $\mathrm{d} M_{t}$, we obtain the different values of the number of shares $\theta_{t}$. That is, we cannot find the number of shares $\theta_{t}$ that lead to the value $V_{T}=h\left(S_{T}\right)=f\left(T, S_{T}\right)$. Thus, we consider an equation given by the term belonging to $\mathrm{d} t$ in (19) and (21). This gives the partial differential equation of the option price.

$$
\begin{aligned}
& r\left(t, S_{t}\right) V_{t}+\theta_{t} S_{t}\left[\mu\left(t, S_{t}\right)-r\left(t, S_{t}\right)+\lambda\left(t, S_{t}\right) \eta_{t}+\kappa\left(t, S_{t}\right) \eta_{t}\right] \\
& =\partial_{t} f\left(t, S_{t}\right)+\left(\mu\left(t, S_{t}\right)+\lambda\left(t, S_{t}\right) \eta_{t}+\kappa\left(t, S_{t}\right) \eta_{t}\right. \\
& \left.\quad-\rho\left[a \sigma\left(t, S_{t}\right)+b \lambda\left(t, S_{t}\right) \zeta_{t}+b \kappa\left(t, S_{t}\right) \zeta_{t}\right]\right) S_{t} \partial_{S} f\left(t, S_{t}\right) \\
& \quad+\frac{1}{2}\left(\sigma\left(t, S_{t}\right)+\lambda\left(t, S_{t}\right) \zeta_{t}+\kappa\left(t, S_{t}\right) \zeta_{t}\right)^{2} S_{t}^{2} \partial_{S S}^{2} f\left(t, S_{t}\right) \\
& \quad+\rho\left(f\left(t, S_{t^{-}}\left(1+a \sigma\left(t, S_{t}\right)+b \lambda\left(t, S_{t}\right) \zeta_{t}+b \kappa\left(t, S_{t}\right) \zeta_{t}\right)\right)-f\left(t, S_{t^{-}}\right)\right)
\end{aligned}
$$

with the terminal condition

$$
f\left(T, S_{T}\right)=h\left(S_{T}\right)
$$

The theorem is proved.

The theorem below shows the finding the number of shares $\theta_{t}$ invested in risky asset in Equation (24).

Theorem 3. The number of shares $\theta_{t}$ that minimizes the distance between the wealth $V_{T}$ and the option price $f\left(T, S_{T}\right)=h\left(S_{T}\right)$ is given by

$$
\begin{aligned}
\theta_{t}= & \frac{(\sigma+\lambda \zeta+\kappa \zeta)^{2} S^{2} \partial_{S} f}{(\sigma+\lambda \zeta+\kappa \zeta)^{2} S^{2}+\rho S^{2}(a \sigma+b \lambda \zeta+b \kappa \zeta)^{2}} \\
& +\frac{\rho S(a \sigma+b \lambda \zeta+b \kappa \zeta)\left(f\left(t, S_{t^{-}}(1+a \sigma+b \lambda \zeta+b \kappa \zeta)\right)-f\right)}{(\sigma+\lambda \zeta+\kappa \zeta)^{2} S^{2}+\rho S^{2}(a \sigma+b \lambda \zeta+b \kappa \zeta)^{2}}
\end{aligned}
$$

Proof. Since we can not find the number of shares $\theta_{t}$ directly. Therefore, in order to find the number of shares $\theta_{t}$ invested in risky asset, we minimize the 
distance between the wealth $V_{T}$ and the option price $f\left(T, S_{T}\right)=h\left(S_{T}\right)$ at time $T$. So, we consider

$$
\min _{\theta} E\left[\left(h\left(S_{T}\right)-V_{T}\right)^{2}\right] .
$$

Applying Ito integral to the wealth process $V_{T}$ in (19), we have

$$
\begin{aligned}
V_{T}= & V_{0}+\int_{0}^{T} r\left(t, S_{t}\right) V_{t}+\theta_{t} S_{t}\left[\mu\left(t, S_{t}\right)-r\left(t, S_{t}\right)+\lambda\left(t, S_{t}\right) \eta_{t}+\kappa\left(t, S_{t}\right) \eta_{t}\right] \mathrm{d} t \\
& +\int_{0}^{T} \theta_{t} S_{t}\left[\sigma\left(t, S_{t}\right)+\lambda\left(t, S_{t}\right) \zeta_{t}+\kappa\left(t, S_{t}\right) \zeta_{t}\right] \mathrm{d} W_{t} \\
& +\int_{0}^{T} \theta_{t} S_{t}\left[a \sigma\left(t, S_{t}\right)+b \lambda\left(t, S_{t}\right) \zeta_{t}+b \kappa\left(t, S_{t}\right) \zeta_{t}\right] \mathrm{d} M_{t} .
\end{aligned}
$$

Similarly for the option price $f\left(T, S_{T}\right)$ in (21), we get

$$
\begin{aligned}
& f\left(T, S_{T}\right) \\
& =f\left(0, S_{0}\right)+\int_{0}^{T}\left[\partial_{t} f\left(t, S_{t}\right)+\left(\mu\left(t, S_{t}\right)+\lambda\left(t, S_{t}\right) \eta_{t}+\kappa\left(t, S_{t}\right) \eta_{t}\right.\right. \\
& \left.-\rho\left[a \sigma\left(t, S_{t}\right)+b \lambda\left(t, S_{t}\right) \zeta_{t}+b \kappa\left(t, S_{t}\right) \zeta_{t}\right]\right) S_{t} \partial_{S} f\left(t, S_{t}\right) \\
& +\frac{1}{2}\left(\sigma\left(t, S_{t}\right)+\lambda\left(t, S_{t}\right) \zeta_{t}+\kappa\left(t, S_{t}\right) \zeta_{t}\right)^{2} S_{t}^{2} \partial_{S S}^{2} f\left(t, S_{t}\right) \\
& \left.+\rho\left(f\left(t, S_{t^{-}}\left(1+a \sigma\left(t, S_{t}\right)+b \lambda\left(t, S_{t}\right) \zeta_{t}+b \kappa\left(t, S_{t}\right) \zeta_{t}\right)\right)-f\left(t, S_{t^{-}}\right)\right)\right] \mathrm{d} t \\
& +\int_{0}^{T}\left(\sigma\left(t, S_{t}\right)+\lambda\left(t, S_{t}\right) \zeta_{t}+\kappa\left(t, S_{t}\right) \zeta_{t}\right) S_{t} \partial_{S} f\left(t, S_{t}\right) \mathrm{d} W_{t} \\
& +\int_{0}^{T}\left(f\left(t, S_{t^{-}}\left(1+a \sigma\left(t, S_{t}\right)+b \lambda\left(t, S_{t}\right) \zeta_{t}+b \kappa\left(t, S_{t}\right) \zeta_{t}\right)\right)-f\left(t, S_{t^{-}}\right)\right) \mathrm{d} M_{t}
\end{aligned}
$$

By (28), (29) and (24), we have

$$
\begin{aligned}
E\left[\left(h\left(S_{T}\right)-V_{T}\right)^{2}\right] \\
=E\left[\left(\int_{0}^{T}\left[\sigma\left(t, S_{t}\right)+\lambda\left(t, S_{t}\right) \zeta_{t}+\kappa\left(t, S_{t}\right) \zeta_{t}\right] S_{t}\left(\partial_{S} f\left(t, S_{t}\right)-\theta_{t}\right) \mathrm{d} W_{t}\right)^{2}\right] \\
+E\left[\left(\int _ { 0 } ^ { T } \left(f\left(t, S_{t^{-}}\left(1+a \sigma\left(t, S_{t}\right)+b \lambda\left(t, S_{t}\right) \zeta_{t}+b \kappa\left(t, S_{t}\right) \zeta_{t}\right)\right)\right.\right.\right. \\
\left.\left.\left.-f\left(t, S_{t^{-}}\right)-\theta_{t} S_{t}\left[a \sigma\left(t, S_{t}\right)+b \lambda\left(t, S_{t}\right) \zeta_{t}+b \kappa\left(t, S_{t}\right) \zeta_{t}\right]\right) \mathrm{d} M_{t}\right)^{2}\right]
\end{aligned}
$$

By Ito isometry [11], we obtain

$$
\begin{aligned}
E & {\left[\left(h\left(S_{T}\right)-V_{T}\right)^{2}\right] } \\
= & \int_{0}^{T} E\left[\left(\left[\sigma\left(t, S_{t}\right)+\lambda\left(t, S_{t}\right) \zeta_{t}+\kappa\left(t, S_{t}\right) \zeta_{t}\right] S_{t}\left(\partial_{S} f\left(t, S_{t}\right)-\theta_{t}\right)\right)^{2}\right] \mathrm{d} t \\
& +\int_{0}^{T} E\left[\rho \left(f\left(t, S_{t^{-}}\left(1+a \sigma\left(t, S_{t}\right)+b \lambda\left(t, S_{t}\right) \zeta_{t}+b \kappa\left(t, S_{t}\right) \zeta_{t}\right)\right)\right.\right. \\
& \left.\left.-f\left(t, S_{t^{-}}\right)-\theta_{t} S_{t}\left[a \sigma\left(t, S_{t}\right)+b \lambda\left(t, S_{t}\right) \zeta_{t}+b \kappa\left(t, S_{t}\right) \zeta_{t}\right]\right)^{2}\right] \mathrm{d} t \\
= & \int_{0}^{T} E\left[l\left(\theta_{t}\right)\right] \mathrm{d} t
\end{aligned}
$$

where 


$$
\begin{aligned}
l\left(\theta_{t}\right)= & (\sigma+\lambda \zeta+\kappa \zeta)^{2} S^{2}\left(\partial_{S} f-\theta_{t}\right)^{2}+\rho\left(f\left(t, S_{t^{-}}(1+a \sigma+b \lambda \zeta+b \kappa \zeta)\right)\right. \\
& \left.-f\left(t, S_{t^{-}}\right)-\theta_{t} S(a \sigma+b \lambda \zeta+b \kappa \zeta)\right)^{2}
\end{aligned}
$$

To find the critical point, we setting $l^{\prime}\left(\theta_{t}\right)=0$, we obtain

$$
\begin{aligned}
\theta_{t}= & \frac{(\sigma+\lambda \zeta+\kappa \zeta)^{2} S^{2} \partial_{s} f}{(\sigma+\lambda \zeta+\kappa \zeta)^{2} S^{2}+\rho S^{2}(a \sigma+b \lambda \zeta+b \kappa \zeta)^{2}} \\
& +\frac{\rho S(a \sigma+b \lambda \zeta+b \kappa \zeta)\left(f\left(t, S_{t^{-}}(1+a \sigma+b \lambda \zeta+b \kappa \zeta)\right)-f\right)}{(\sigma+\lambda \zeta+\kappa \zeta)^{2} S^{2}+\rho S^{2}(a \sigma+b \lambda \zeta+b \kappa \zeta)^{2}}
\end{aligned}
$$

By second derivative test, we obtain

$$
l^{\prime \prime}\left(\theta_{t}\right)=2(\sigma+\lambda \zeta+\kappa \zeta)^{2} S^{2}+2 \rho S^{2}(a \sigma+b \lambda \zeta+b \kappa \zeta)^{2}>0
$$

thus, the function has a minimum at critical point. Hence, we have the Theorem.

Note from the above result that if there is no jump (i.e. $a=b=0$ ), we have

$$
\theta_{t}=\partial_{s} f
$$

and the PDE of the option price in Equation (24) is reduced to the equation obtained in [9]. Similarly, If the no transaction cost assumption is assumed (i.e. $\left.\kappa\left(t, S_{t}\right)=0\right)$, the Equation (31) becomes

$$
\theta_{t}=\frac{(\sigma+\lambda \zeta)^{2} S^{2} \partial_{S} f+\rho S(a \sigma+b \lambda \zeta)\left(f\left(t, S_{t^{-}}(1+a \sigma+b \lambda \zeta)\right)-f\right)}{(\sigma+\lambda \zeta)^{2} S^{2}+\rho S^{2}(a \sigma+b \lambda \zeta)^{2}}
$$

and the Equation (24) is reduced to a form as in [8]. The Black-Scholes model is obtained when $a=b=\kappa\left(t, S_{t}\right)=\lambda\left(t, S_{t}\right)=0$.

\section{Conclusion}

In this paper, a new differential form of assets price is introduced and a European option pricing model with transaction costs and jumps in an illiquid market is derived. This extends the assumptions of the Black-Scholes model by combining the jump-diffusion and existence of transaction costs in illiquid markets. This approach might be more realistic to the financial market.

\section{Acknowledgements}

The authors appreciate referee(s) for their helpful comments. The first authors would like to thank the Department of Mathematics, Faculty of Science, King Mongkut's University of Technology Thonburi for financial support.

\section{Conflicts of Interest}

The authors declare no conflicts of interest regarding the publication of this paper.

\section{References}

[1] Black, F. and Scholes, M. (1973) The Pricing of Options and Corporate Liabilities. 
Journal of Political Economy, 81, 637-654. https://doi.org/10.1086/260062

[2] Dokuchaev, N.G. and Savkin, A.V. (1998) The Pricing of Options in a Financial Market Model with Transaction Costs and Uncertain Volatility. Journal of Multinational Financial Management, 8, 353-364. https://doi.org/10.1016/S1042-444X(98)00036-X

[3] Florescu, I., Mariant, M.C. and Sengupta, I. (2014) Option Pricing with Transaction Costs and Stochastic Volatility. Electronic Journal of Differential Equations, 2014, $1-19$.

[4] FEdeki, S.O., Ugbebor, O.O. and Owoloko, E.A. (2017) On a Nonlinear Transaction-Cost Model for Stock Prices in an Illiquid Market Driven by a Relaxed Black-Scholes Model Assumption. Malaysian Journal of Mathematical Sciences, 11, 83-96.

[5] Liu, H. and Yong, J. (2005) Option Pricing with an Illiquid Asset Market. Journal of Economic Dynamics \& Control, 29, 2125-2156. https://doi.org/10.1016/j.jedc.2004.11.004

[6] Glover, K.J., Duck, P.W. and Newton, D.P. (2010) On Nonlinear Models of Markets with Finite Liquidity: Some Cautionary Notes. SIAM Journal on Applied Mathematics, 70, 3252-3271. https://doi.org/10.1137/080736119

[7] Pirvu, T.A. and Yazdanian, A. (2015) Numerical Analysis for Spread Option Pricing Model in Illiquid underlying Asset Market: Full Feedback Model. Applied Mathematics \& Information Sciences, 1271-1281. https://doi.org/10.18576/amis/100406

[8] El-Khatib, Y. and Hatemi-J, A. (2013) On Option Pricing in Illiquid Markets with Jumps. International Scholarly Research Notices, 2013, Article ID 567071. https://doi.org/10.1155/2013/567071

[9] Agana, F., Makinde, O.D. and Theuri, D.M. (2016) Numerical Treatment of a Generalized Black-Scholes Model for Options Pricing in an Illiquid Financial Market with Transections Costs. Global Journal of Pure and Applied Mathematics, 12, 4349-4361.

[10] Rouah, F. (2013) Four Derivations of the Black Scholes PDE. http://frouah.com/finance\%20notes/Black\%20Scholes\%20PDE.pdf

[11] Mukam, J.D. (2015) Stochastic Calculus with Jumps Processes: Theory and Numerical Techniques. M.S. Dissertation, African Institute for Mathematical Sciences (AIMS) Senegal. 